This is an electronic reprint of the original article. This reprint may differ from the original in pagination and typographic detail.

Author(s): Kotila, Jenni-Mari; Barea, J.

Title: Occupation probabilities of single particle levels using the microscopic interacting boson model: Application to some nuclei of interest in neutrinoless double- $\beta$ decay

Year: $\quad 2016$

Version:

Please cite the original version:

Kotila, J.-M., \& Barea, J. (2016). Occupation probabilities of single particle levels using the microscopic interacting boson model: Application to some nuclei of interest in neutrinoless double- $\beta$ decay. Physical Review C, 94(3), Article 034320.

https://doi.org/10.1103/PhysRevC.94.034320

All material supplied via JYX is protected by copyright and other intellectual property rights, and duplication or sale of all or part of any of the repository collections is not permitted, except that material may be duplicated by you for your research use or educational purposes in electronic or print form. You must obtain permission for any other use. Electronic or print copies may not be offered, whether for sale or otherwise to anyone who is not an authorised user. 


\title{
Occupation probabilities of single particle levels using the microscopic interacting boson model: Application to some nuclei of interest in neutrinoless double- $\beta$ decay
}

\author{
J. Kotila ${ }^{1,2, *}$ and J. Barea ${ }^{3, \dagger}$ \\ ${ }^{1}$ University of Jyväskylä, Department of Physics, P.O. Box 35, FI-40014 Jyväskylä, Finland \\ ${ }^{2}$ Center for Theoretical Physics, Sloane Physics Laboratory, Yale University, New Haven, Connecticut 06520-8120, USA \\ ${ }^{3}$ Departamento de Física, Universidad de Concepción, Casilla 160-C, Concepción 4070386, Chile \\ (Received 11 January 2016; revised manuscript received 1 August 2016; published 21 September 2016)
}

\begin{abstract}
We have developed a new method to calculate the occupancies of single particle levels in atomic nuclei. This method has been developed in the context of the microscopic interacting boson model, in which neutron and proton degrees of freedom are treated explicitly. The energies of the single particle levels constitute a very important input for the calculation of the occupancies in this method. In principle these energies can be considered as input parameters that can be fitted to reproduce the experimental occupancies. Instead of fitting, in this study we have extracted the single particle energies from experimental data on nuclei with a particle more or one particle less than a shell closure. We provide the sets of these single particle energies suitable for several major shells and apply our method to calculate the occupancies of several nuclei of interest in neutrinoless double- $\beta$ decay using these sets. Our results are compared with other theoretical calculations and experimental occupancies, when available.
\end{abstract}

DOI: 10.1103/PhysRevC.94.034320

\section{INTRODUCTION}

In models of nuclear structure, the energies of the single particle levels play a very important role. They are crucial tests of the shell model for semimagic or doubly magic nuclei, but also become essential input parameters in the (interacting) shell model (ISM) or quasiparticle random-phase approximation (QRPA) calculations or any other model where single particle degrees of freedom have to be considered. These energies change across the periodic table due to the residual proton-neutron interaction among valence nucleons. Actually they are modulated by the monopole proton-neutron interaction in the language of the shell model. Then they shed light about this interaction directly. Indirectly they are also of interest in astrophysical calculations. For instance, the calculation of the abundances in the $r$-process nucleosynthesis for medium and heavy nuclei depends strongly on the $\beta$-decay half-lives and branchings for $\beta$-delayed neutron emission $P_{n}$ values of nuclei present in the $r$-process path. Both quantities have to be provided by some nuclear structure model and their calculation depends again on the single particle levels and their energies.

One concept intimately related to the single particle levels is their occupancies. Experimentally ground-state occupancies can be obtained by one-nucleon transfer reactions, pick-up or stripping, offering an important test for the theoretical models used to calculate nuclear properties. Through the analysis of the experimental spectroscopic factors of stripping and pick-up reactions the occupation numbers of the orbits close to the Fermi level can be extracted. This has been recently done, or is under investigation at the moment, for neutrons or for both neutrons and protons in ${ }^{76} \mathrm{Ge},{ }^{76} \mathrm{Se},{ }^{100} \mathrm{Mo}$,

\footnotetext{
*jenni.kotila@jyu.fi

†jbarea@udec.cl
}

${ }^{100} \mathrm{Ru},{ }^{130} \mathrm{Te},{ }^{130} \mathrm{Xe},{ }^{136} \mathrm{Xe},{ }^{136} \mathrm{Ba},{ }^{150} \mathrm{Nd}$, and ${ }^{150} \mathrm{Sm}$ in a series of very careful experiments [1-5]. These nuclei are candidates to participate in neutrinoless double- $\beta$ decay and recently the role of the chosen single-particle valence space and orbital occupancies has also become one of the central issues in the calculation of the double- $\beta$-decay nuclear matrix elements (NMEs) [1,2,6-8]. As major experimental efforts are being made in the search for massive Majorana neutrinos and their absolute mass scale through the process of neutrinoless double- $\beta$ decay, the calculation of these NMEs is an essential step in the study of $0 \nu \beta \beta$ decay, which is crucial for extracting the neutrino mass from experimental half-life. The fact that $0 \nu \beta \beta$ decay is a unique process, and there is no direct probe which connects the initial and final states other than the process itself, makes the prediction challenging for theoretical models. Thus, the calculation of the occupancies of the single particle levels would allow one to satisfy a twofold goal: to assess the goodness of the single particle energies and to check the reliability of the used wave functions. Both tests are particularly important in the case of nuclei involved in double- $\beta$ decay, because they affect the evaluation of the NMEs and then their reliability [9].

The main purpose of this article is to show a new method to calculate occupancies in the framework of the microscopic interacting boson model, also known as IBM-2 [10]. For that purpose the single particle energies are one of the main inputs. We devote an entire section to explain how we choose their values. Next we apply our method to a set of nuclei that we have studied in our previous articles, where phase space factors and prefactors [11-13] and NMEs in IBM-2 [13-18] needed for the theoretical description of $0 v \beta^{-} \beta^{-}, 2 v \beta^{-} \beta^{-}, 0 v \beta^{+} \beta^{+}, 0 v \beta^{+} E C+, R 0 v E C E C$, $2 v \beta^{+} \beta^{+}, 2 v \beta^{+} E C$, and $2 v E C E C$ decay mediated in the case of neutrinoless decay by light or heavy neutrino exchange, as well as for the Majoron emitting modes of $0 \nu \beta^{-} \beta^{-}$decay [19], and the limits on sterile neutrino contributions to neutrinoless 
double- $\beta$ decay [20] were calculated. Previous studies about the impact of the experimental analysis of the occupancies on the different calculations show that the experimental results were in reasonable agreement with the ISM ones [8,21], while the agreement was unsatisfactory with the QRPA ones [22,23]. Furthermore, when the QRPA calculations were modified to reproduce the experimental data, the double- $\beta$-decay NMEs came closer to the ISM one. Our theoretical predictions are compared with these models along with experimental data when available.

The article is organized as follows: In Sec. II we describe the theoretical approach for the calculation of orbital occupancies in IBM-2. Secion III is devoted to explaining how we chose the single particle energies from experimental data. In Sec. IV the results of our calculations are analyzed and compared with those from other models and with experimental data, when available. Conclusions are drawn in Sec. V.

\section{THEORETICAL DESCRIPTION}

The mapping procedure of the shell model into the microscopic IBM is described in more detail in Refs. [24,25] and more recently in Ref. [14]. Basically, the shell-model creation operators of collective pairs of protons $(\rho=\pi)$ and neutrons ( $\rho=v$ ) of angular momenta 0 and 2 , the $S$ and $D$ pairs,

$$
\begin{aligned}
S_{\rho}^{\dagger} & =\sum_{j} \alpha_{\rho, j} \sqrt{\frac{\Omega_{j}}{2}}\left(\rho_{j}^{\dagger} \times \rho_{j}^{\dagger}\right)^{(0)}, \\
D_{\rho, M}^{\dagger} & =\sum_{j \leqslant j^{\prime}} \beta_{\rho, j j^{\prime}} \frac{1}{\sqrt{1+\delta_{j j^{\prime}}}}\left(\rho_{j}^{\dagger} \times \rho_{j^{\prime}}^{\dagger}\right)_{M}^{(2)},
\end{aligned}
$$

with $\Omega_{j}=j+1 / 2$, are used to span the $S D$ Fermion spaces, which are subspaces of the full shell-model spaces for each kind of nucleon. Here the generalized seniority $v$ is a quantum number used to label the states of each subspace as $|n, v, \alpha, J\rangle$, where $n$ indicates the number of protons or neutrons, $J$ is the angular momentum, and $\alpha$ contains all further quantum numbers required to uniquely specify the state. The pair structure coefficients $\alpha_{\rho, j}$ and $\beta_{\rho, j j^{\prime}}$ can be obtained in different ways [26-31]. Here we followed the method described in Ref. [31], in which $S_{\rho}^{\dagger}$ and $D_{\rho, M}^{\dagger}$ create the energetically lowest $0^{+}$and $2^{+}$two-fermion states appropriate to a nucleus with two particles or two holes outside a closed shell. By using this method some possible renormalization (polarization) effects induced by the neutron-proton interaction are included

TABLE I. Single particle energies and isovector SDI strength parameters $A_{1}$ in $\mathrm{MeV}$ used for the $28-50$ shell.

\begin{tabular}{lccc}
\hline \hline Orbital & $\begin{array}{c}\text { Protons } \\
\text { (particles) } \\
A \sim 76\end{array}$ & $\begin{array}{c}\text { Protons } \\
\text { (holes) } \\
A \sim 0.299\end{array}$ & $\begin{array}{c}\text { Neutrons } \\
\text { (holes) }\end{array}$ \\
& $A_{1}=0.239$ & $\begin{array}{c}A \sim 76 \\
A_{1}=0.237\end{array}$ \\
\hline $2 p_{1 / 2}$ & 1.179 & 0.678 & 0.588 \\
$2 p_{3 / 2}$ & 0.000 & 1.107 & 1.095 \\
$1 f_{5 / 2}$ & 0.340 & 1.518 & 1.451 \\
$1 g_{9 / 2}$ & 2.640 & 0.000 & 0.000 \\
\hline \hline
\end{tabular}

TABLE II. Single particle energies and isovector SDI strength parameters $A_{1}$ in $\mathrm{MeV}$ used for the 50-82 shell.

\begin{tabular}{lcccc}
\hline \hline Orbital & $\begin{array}{c}\text { Protons } \\
\text { (particles) } \\
A \sim 130 \\
A_{1}=0.222\end{array}$ & $\begin{array}{c}\text { Protons } \\
\text { (particles) } \\
A \sim 150\end{array}$ & $\begin{array}{c}\text { Neutrons } \\
\text { (particles) }\end{array}$ & $\begin{array}{c}\text { Neutrons } \\
\text { (holes) }\end{array}$ \\
& $A_{1}=0.223$ & $A_{1}=0.242$ & $\begin{array}{c}A \sim 130 \\
A_{1}=0.163\end{array}$ \\
\hline $3 s_{1 / 2}$ & 2.990 & 0.719 & 0.775 & 0.332 \\
$2 d_{3 / 2}$ & 2.440 & 0.466 & 1.142 & 0.000 \\
$2 d_{5 / 2}$ & 0.962 & 0.365 & 0.000 & 1.654 \\
$1 g_{7 / 2}$ & 0.000 & 0.000 & 0.172 & 2.434 \\
$1 h_{11 / 2}$ & 2.792 & 0.668 & 2.868 & 0.069 \\
\hline \hline
\end{tabular}

approximately. The surface delta interaction (SDI) is chosen as the effective interaction between identical nucleons using an isovector strength parameter $A_{1}$ whose value is fitted to reproduce the energy difference between the first $2^{+}$state and the $0^{+}$ground state in the corresponding two-valence-particle or two-valence-hole nucleus.

The obtained pair structure coefficients are normalized as

$$
\begin{aligned}
\sum_{j} \Omega_{j} \alpha_{j}^{2} & =\sum_{j} \Omega_{j}, \\
\sum_{j \leqslant j^{\prime}} \beta_{j j^{\prime}}^{2} & =1,
\end{aligned}
$$

where from now on we omit the $\rho$ label for convenience.

The states of the $S D$ subspaces are mapped onto boson states belonging to the IBM space, and the boson image of the shell-model number operator at leading order is

$$
\hat{n}_{j}^{B}=A(j)\left(s^{\dagger} \cdot s\right)+B(j)\left(d^{\dagger} \cdot \tilde{d}\right),
$$

where the coefficients $A(j)$ and $B(j)$ are obtained using the OAI mapping procedure of Otsuka, Arima, and Iachello [25], in which the matrix elements of $\hat{n}_{j}^{B}$ between IBM states are made equal to the corresponding shell-model matrix elements

$$
\begin{aligned}
\left\langle s^{\frac{n}{2}}\left\|\hat{n}_{j}^{B}\right\| s^{\frac{n}{2}}\right\rangle & =\left\langle n, 0,0\left\|\hat{n}_{j}\right\| n, 0,0\right\rangle, \\
\left\langle s^{\frac{n}{2}-1} d\left\|\hat{n}_{j}^{B}\right\| s^{\frac{n}{2}-1} d\right\rangle & =\left\langle n, 2,2\left\|\hat{n}_{j}\right\| n, 2,2\right\rangle .
\end{aligned}
$$

The commutator method introduced by Frank and Van Isacker [32] and by Lipas et al. [33] can be used as a general method for calculating exactly matrix elements in the shell

TABLE III. Single particle energies and isovector SDI strength parameters $A_{1}$ in $\mathrm{MeV}$ used for the 82-126 shell.

\begin{tabular}{lcc}
\hline \hline Orbital & $\begin{array}{c}\text { Neutrons } \\
\text { (particles) } \\
A \sim 130 \\
A_{1}=0.133\end{array}$ & $\begin{array}{c}\text { Neutrons } \\
\text { (particles) }\end{array}$ \\
& 1.363 & $A \sim 150$ \\
$A_{1}=0.110$ \\
\hline $3 p_{1 / 2}$ & 0.854 & 1.847 \\
$3 p_{3 / 2}$ & 2.005 & 1.152 \\
$2 f_{5 / 2}$ & 0.000 & 1.948 \\
$2 f_{7 / 2}$ & 1.561 & 0.000 \\
$1 h_{9 / 2}$ & 3.700 & 1.397 \\
$1 i_{13 / 2}$ & & 0.997 \\
\hline \hline
\end{tabular}



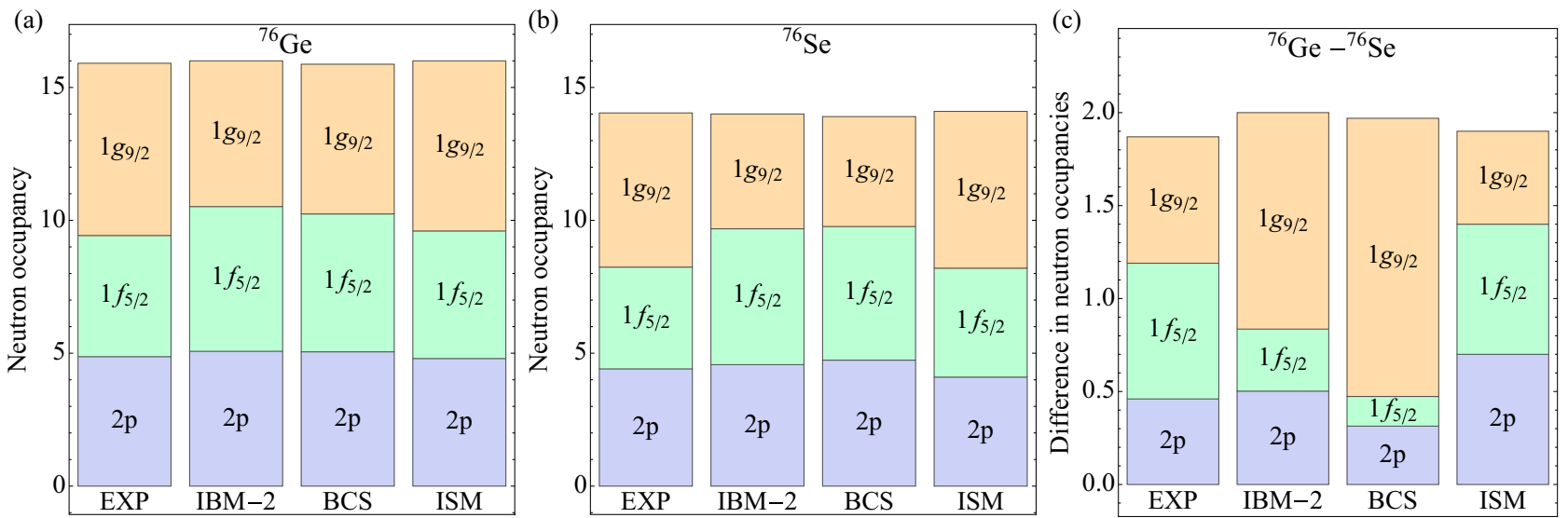

FIG. 1. IBM-2 occupations in (a) ${ }^{76} \mathrm{Ge}$, (b) ${ }^{76} \mathrm{Se}$, and (c) their change for neutrons, compared with experimental [1,2], BCS [22,42], and ISM $[21,43]$ results.

model using the generalized seniority scheme. Noting that $N=n / 2$ is the number of pairs (bosons in the IBM), we finally obtain

$$
\begin{aligned}
A(j)= & \frac{1}{N}\left\langle 2 N, 0,0\left\|\hat{n}_{j}\right\| 2 N, 0,0\right\rangle \\
B(j)= & \frac{1}{\sqrt{5}}\left\langle 2 N, 2,2\left\|\hat{n}_{j}\right\| 2 N, 2,2\right\rangle \\
& -\left(1-\frac{1}{N}\right)\left\langle 2 N 00\left\|\hat{n}_{j}\right\| 2 N 00\right\rangle .
\end{aligned}
$$

The fact that $\hat{n}=\sum_{j} \hat{n}_{j}$ gives the total number of valence nucleons imposes the following sum rules for $A(j)$ and $B(j)$ :

$$
\sum_{j} A(j)=\sum_{j} B(j)=2 .
$$

The orbital occupancies are then obtained from the matrix elements of $\hat{n}_{j}^{B}$ between the realistic wave functions obtained from IBM-2, which in addition to spherical nuclei are also capable of describing medium and heavy deformed nuclei like ${ }^{150} \mathrm{Nd}$ and ${ }^{150} \mathrm{Sm}$. The values of the Hamiltonian parameters used to calculate wave functions of different nuclei can be found in Ref. [16]. These values were obtained from phenomenological fits to spectroscopic data and are unrelated to the single particle energies used in this work, but it is possible to obtain the Hamiltonian parameters on a completely microscopic basis using the single particle energies as it was done in Ref. [31].

\section{SINGLE PARTICLE ENERGIES}

The single particle energies enter the calculation through one-body terms in the two-particle (two-hole) Hamiltonian used to extract the $\alpha$ and $\beta$ pair structure coefficients. We proceed to explain the origin of the different sets of energies we have used in this work, classified according to the major shell where they belong.

\section{A. Shell 28-50}

The single particle energies for protons (particles or holes) and neutrons (holes) in the orbitals of the 28-50 shell are shown in Table I. For proton particles they are suitable for $A \sim 76$ and were obtained from a linear interpolation between the single particle energies extracted from ${ }^{57} \mathrm{Cu}$ (second column in Table XI in Ref. [14]), suitable for $A \sim 56$, and the single particle energies extracted from isotones $N=50$ (third column in Table XI in Ref. [14], but inverted to use (a)

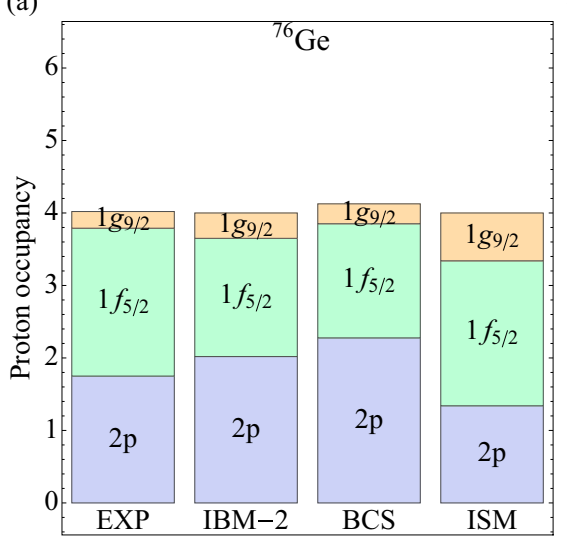

(b)

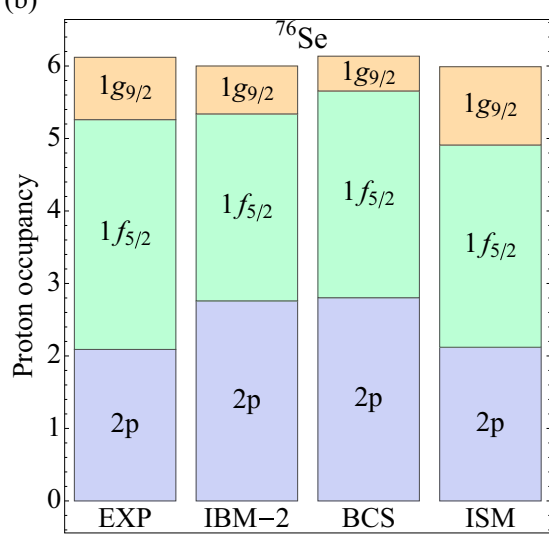

(c)

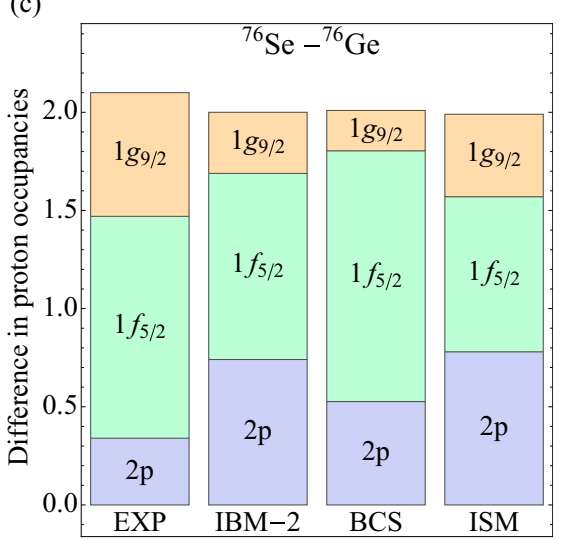

FIG. 2. Same as Fig. 1 for protons. 
(a)

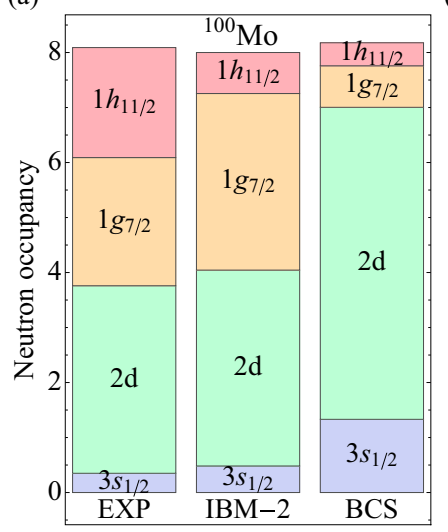

(b)

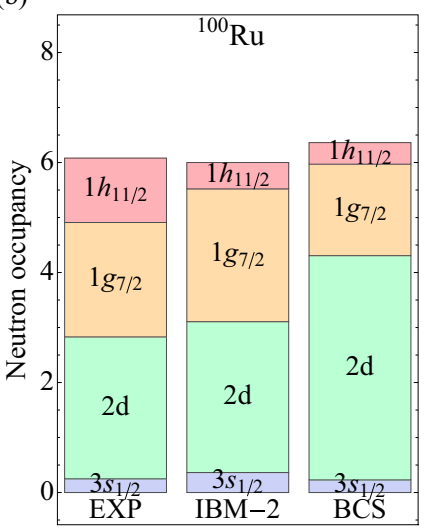

(c)

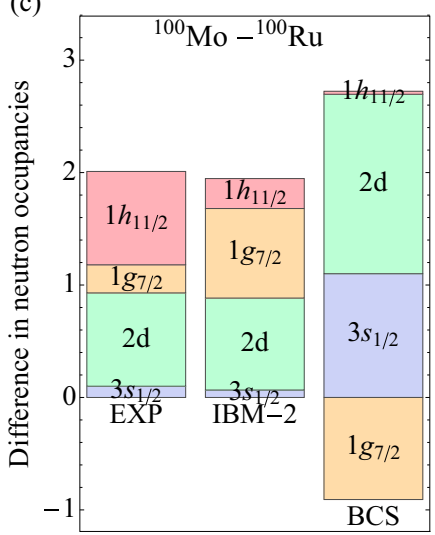

FIG. 3. IBM-2 occupations in (a) ${ }^{100} \mathrm{Mo}$, (b) ${ }^{100} \mathrm{Ru}$, and (c) their change for neutrons, compared with experimental [4] and BCS [7,42] results.

particle and not hole energies), suitable for $A \sim 100$ and neutron number $N<50$. The isovector SDI strength $A_{1}$, assuming a linear dependence in $A^{-1}$, was also interpolated between the value $A_{1}=0.35$ suitable for $A \sim 56$, which reproduces the excitation energy of the $2_{1}^{+}$state in ${ }^{58} \mathrm{Zn}$, and the value $A_{1}=0.264$ suitable for $A \sim 100$, which reproduces the excitation energy of the $2_{1}^{+}$state in ${ }^{98} \mathrm{Cd}$. The single particle energies for proton holes were extracted from the spectrum of ${ }^{107}$ In, suitable for $A \sim 100$ and $N \sim 60$. The strength $A_{1}$ was calculated with these single particle energies to reproduce the excitation energy of the $2_{1}^{+}$state in ${ }^{98} \mathrm{Cd}$. The single particle energies for neutron holes were extracted from the spectrum of ${ }^{89} \mathrm{Zr}$, suitable for $A \sim 90$ and $Z \sim 40$. The strength $A_{1}$ was calculated with these single particle energies to reproduce the excitation energy of the $2_{1}^{+}$state in ${ }^{98} \mathrm{Cd}$.

\section{B. Shell 50-82}

The single particles energies for protons and neutrons (particle or hole) in the orbitals of the 50-82 shell are shown in Table II. The energies for proton particles were extracted from the excitation spectrum of ${ }^{133} \mathrm{Sb}$, except for the $3 s_{1 / 2}$ level, whose energy was taken from systematics in odd nuclei with $N=82$ [34]. The isovector SDI strength $A_{1}$ was fitted to reproduce the excitation energy of the $2_{1}^{+}$state in ${ }^{134} \mathrm{Te}$. We have also considered an alternative set of proton particle energies suitable for $A=150$ based on the assumption of a subshell closure at $Z=64$ [35-40]. They were extracted from the excitation spectrum of ${ }^{147} \mathrm{~Tb}$. In this case the strength $A_{1}$ was obtained to reproduce the excitation energy of the $2_{1}^{+}$state in ${ }^{148} \mathrm{Dy}$. The energies of the orbitals $1 g_{7 / 2}, 3 s_{1 / 2}$, and $2 d_{3 / 2}$ for neutron particles were extracted from the excitation spectra of ${ }^{101} \mathrm{Sn},{ }^{97} \mathrm{Pd}$, and ${ }^{95} \mathrm{Ru}$, respectively, while the energy of the orbital $1 h_{11 / 2}$ was obtained from systematics in odd nuclei with $N=51$. For this case, the strength $A_{1}$ was obtained to reproduce the excitation energy of the $2_{1}^{+}$state in ${ }^{102} \mathrm{Sn}$. The energies for neutron holes were extracted from the excitation spectrum of ${ }^{131} \mathrm{Sn}$ and the corresponding strengths $A_{1}$ from a fit to the excitation energy of the $2_{1}^{+}$state in ${ }^{130} \mathrm{Sn}$.

\section{Shell 82-126}

The single particle energies for neutron particles are shown in Table III. We present two sets. The first one was obtained from the excitation spectrum of ${ }^{133} \mathrm{Sn}$ and the strength $A_{1}$ from a fit to the excitation energy of the $2_{1}^{+}$state in ${ }^{134} \mathrm{Sn}$. The second one was obtained from the excitation spectrum of ${ }^{147} \mathrm{Gd}$ and the strength $A_{1}$ from a fit to the excitation energy of the $2_{1}^{+}$ state in ${ }^{148} \mathrm{Gd}$, assuming a shell gap at $Z=64$.

\section{RESULTS}

\section{A. $A=76$ nuclei}

In the case of ${ }^{76} \mathrm{Ge}$ and ${ }^{76} \mathrm{Se}$ both neutron vacancies and proton occupancies have been determined recently by accurate measurements of one-nucleon transfer reactions [1,2]. The theoretical IBM-2 occupancies are shown in Figs. 1 and 2, for neutrons and protons, respectively, along with the experimental, QRPA at BCS level [22,41,42], and ISM [21,43] results. As one can see from Fig. 1, compared to experimental data, $2 p$ and $1 f_{5 / 2}$ orbitals are overfilled, leaving the $1 g_{9 / 2}$ orbital underfilled for both ${ }^{76} \mathrm{Ge}$ and ${ }^{76} \mathrm{Se}$. The change in occupancy in IBM2 is then dominated by the $1 g_{9 / 2}$ orbital, while experimentally the $1 g_{9 / 2}$ and $1 f_{5 / 2}$ orbitals give roughly equal contributions. Compared to other theoretical calculations IBM-2 results deviate more from measurements than the ISM values but are in better correspondence than the BCS calculation.

In the case of proton occupancies (Fig. 2), a depletion of protons in the $1 f_{5 / 2}$ orbital is seen for both ${ }^{76} \mathrm{Ge}$ and ${ }^{76} \mathrm{Se}$ when comparing IBM-2 results to experiments. However, when looking at the change in proton occupancies, the IBM-2 results gives slightly better agreement with experiments than the ISM results.

One should note that in the IBM-2 calculation the same single particle energies and SDI strength parameters for both initial and final states are used, whereas in the BCS calculation rather different sets of single particle energies are employed. In addition, the wave functions used in this work lack for some components from configuration mixing calculations, which is widely accepted in the IBM community as necessary in this region of the nuclear chart [44]. 

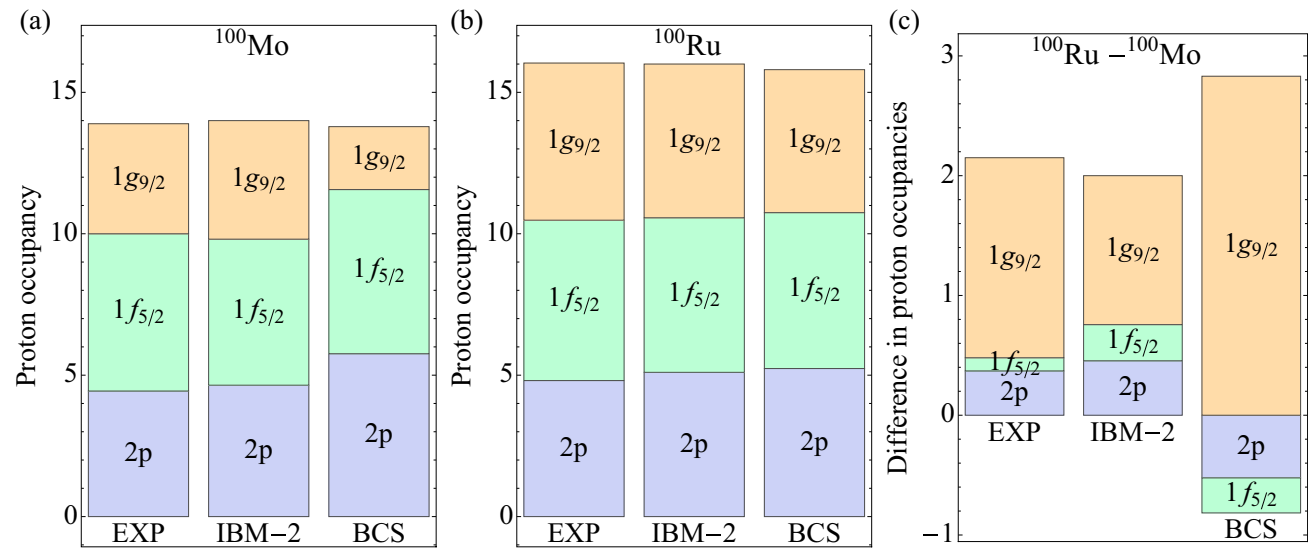

FIG. 4. Same as Fig. 3 for protons.

\section{B. $A=100$ nuclei}

The calculated occupancies for ${ }^{100} \mathrm{Mo}$ and ${ }^{100} \mathrm{Ru}$ are shown and compared with experimental [4] and BCS [7,42] results in Figs. 3 and 4. For neutrons (Fig. 3), the change appears to be dominated by the $2 d$ orbitals in good agreement with experiments. Experimental data show a large contribution also from the $1 h_{11 / 2}$ orbital and a smaller contribution from the $1 g_{7 / 2}$ orbital, but these strengths seem to be somewhat switched in the IBM-2 prediction. The predicted small contribution from the $3 s_{1 / 2}$ orbital coincides well with experiments. BCS calculation, however, predicts a more complex rearrangement of nucleons that differs both from experiments and IBM-2 results.

For protons (Fig. 4), the individual ${ }^{100} \mathrm{Mo}$ and ${ }^{100} \mathrm{Ru}$ proton occupancies, as well as the difference in proton occupancy, are in proper agreement with the experiments. The change is dominated by the $1 g_{9 / 2}$ orbital, whereas $2 p$ orbitals play a lesser role and the $1 f_{5 / 2}$ orbital gives only a small contribution. Also in this case comparison with BCS calculations reveals complex differences.

\section{C. $A=128$ and 130 nuclei}

Experimental study of the $A=130$ nuclei was recently reported in Refs. [3,5], where also the neutron vacancies and proton occupancies for ${ }^{128} \mathrm{Te}$ were given. The corresponding neutron occupancies are shown in Figs. 5 and 6, respectively. Comparing the calculated and experimental orbital occupancies of Fig. 5 one notices that the overall agreement in panels (a) and (b) is good for all the theoretical calculations compared to experimental data. When looking at Fig. 5(c), the difference in neutron occupancies, the $1 h_{11 / 2}$ orbital dominates in the calculations, while experimentally the $2 d$ orbitals are dominant. Furthermore, in all calculations the $1 g_{7 / 2}$ orbital is somewhat depleted while measured the orbital is full. When comparing the different calculations one notices that IBM-2 predicts the smallest changes in $1 g_{7 / 2}$ and $3 s_{1 / 2}$ orbitals, in best agreement with the experiments.

From Fig. 6 one notices that also in the case of $A=128$ nuclei the predicted neutron vacancy in orbital $1 g_{7 / 2}$ has not been experimentally confirmed. Besides that, the agreement in Fig. 6(a) is good for all theoretical calculations with experiments. For ${ }^{128} \mathrm{Xe}$, and thus also for the difference in neutron occupancies, there are no experimental data, but instead we can compare our results with BCS calculations. The agreement is found to be good both for the ${ }^{128} \mathrm{Xe}$ neutron occupancy and for the difference in neutron occupancies. The largest contribution is coming from the $1 h_{11 / 2}$ orbital, followed by the $2 d$ orbitals. (a)

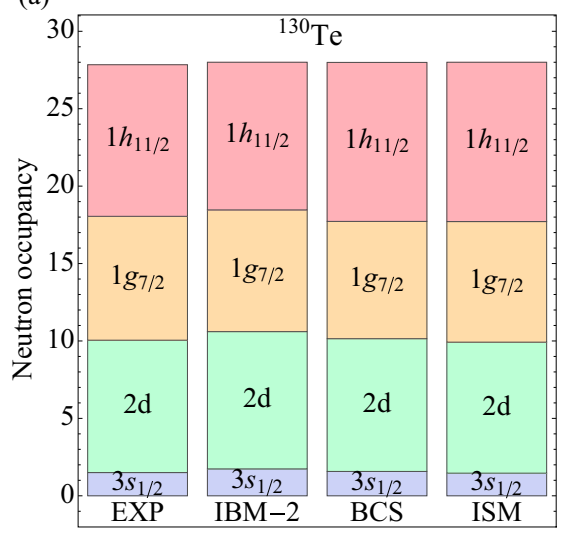

(b)

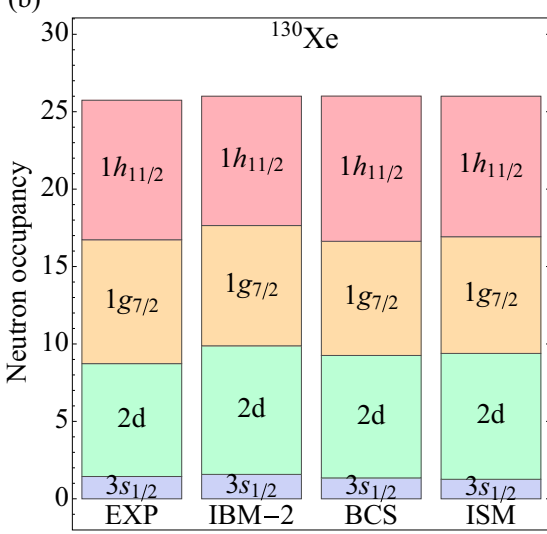

(c)

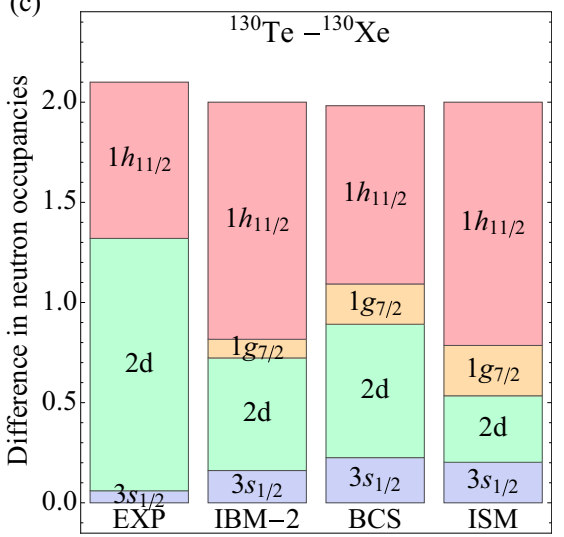

FIG. 5. IBM-2 occupations in (a) ${ }^{130} \mathrm{Te}$, (b) ${ }^{130} \mathrm{Xe}$, and (c) their change for neutrons, compared with experimental [3], BCS [41], and ISM [8] results. 
(a)

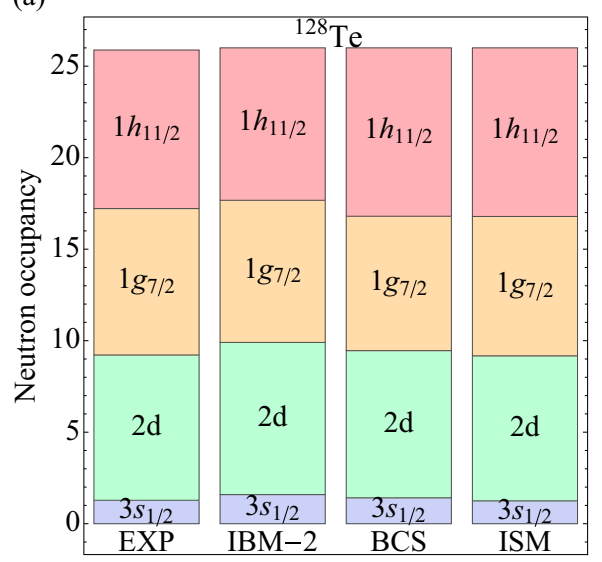

(b)

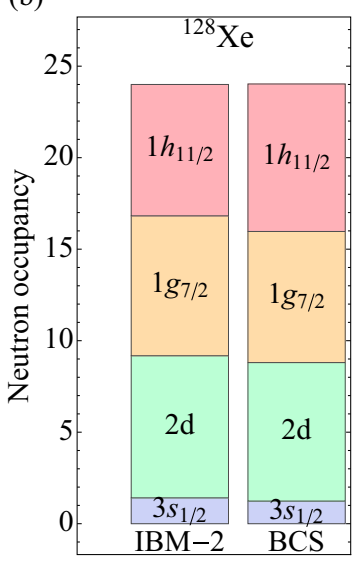

(c)

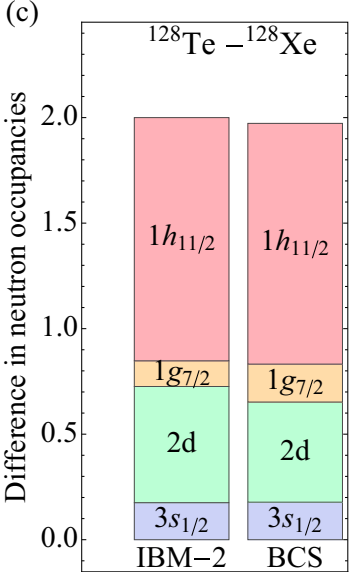

FIG. 6. Same as Fig. 5 for ${ }^{128} \mathrm{Te}$ and ${ }^{128} \mathrm{Xe}$.

In the case of the proton occupancies (Fig. 7), there is a good agreement in ${ }^{130} \mathrm{Te}$ between the experimental data and the calculations, except for the BCS calculations. This comparison fails in the case of ${ }^{130} \mathrm{Xe}$ and this is explicitly shown in the difference in proton occupancies. The ISM overestimates the $2 d$ orbitals and underestimates the $1 g_{7 / 2}$ orbital, while the IBM-2 calculation does the opposite. The BCS calculation is sort of in between. For ${ }^{128} \mathrm{Te}$ (Fig. 8) the results are similar to those of ${ }^{130} \mathrm{Te}$ : the IBM-2 and ISM calculations reproduce well the occupancies, while the BCS calculation shows some discrepancies. However, there are no accurate experimental data available for ${ }^{128} \mathrm{Xe}$, and for the moment our results are compared only with the BCS calculation [41,42]. Both the IBM-2 and BCS calculations predict the $1 g_{7 / 2}$ orbital's dominance over the $2 d$ orbitals, but the BCS calculation also gives a strong contribution from the $2 d$ orbitals.

\section{D. $A=136$ nuclei}

In the case of $A=136$ nuclei, new experimental data for protons are available [5]. Figure 9 shows that in general IBM-2 reproduces well the data, where $1 g_{7 / 2}$ is the most filled orbital. In ${ }^{136} \mathrm{Xe}$ the occupancy of the $2 d$ orbitals is slightly overestimated, while the opposite happens in ${ }^{136} \mathrm{Ba}$ and also for the $1 g_{7 / 2}$ orbital. This small difference produces the discrepancies between experimental and calculated differences in proton occupancies. For neutrons ${ }^{136} \mathrm{Xe}$ is magic and for

${ }^{136} \mathrm{Ba}$ experimental data are unavailable. In this case our calculations predict roughly equal contributions to the $2 d$ and $1 h_{11 / 2}$ orbitals.

\section{E. $A=150$ nuclei}

The nuclei with $A=150$ are very interesting because there is a change in deformation between the initial and final states. Also the location of the Fermi level may be different for ${ }^{150} \mathrm{Nd}$ and ${ }^{150} \mathrm{Sm}$. However, in our calculations, for now, we have used the same single particle energies and isovector strengths for both the initial and final states, as previously mentioned in the discussion of $A=76$ nuclei. In addition, we have performed two different calculations. The first one uses single particle levels from ${ }^{133} \mathrm{Sb}$ for protons and ${ }^{133} \mathrm{Sn}$ for neutrons, which we refer to as Set I, and they are quoted in Tables II and III, respectively, under the columns labeled with $A \sim 130$. The second calculation uses single particle levels from ${ }^{147} \mathrm{~Tb}$ for protons and ${ }^{147} \mathrm{Gd}$ for neutrons, which we refer to as Set II, and they are quoted in Tables II and III, respectively, under the columns labeled with $A \sim 150$. The results obtained with Set I (a)

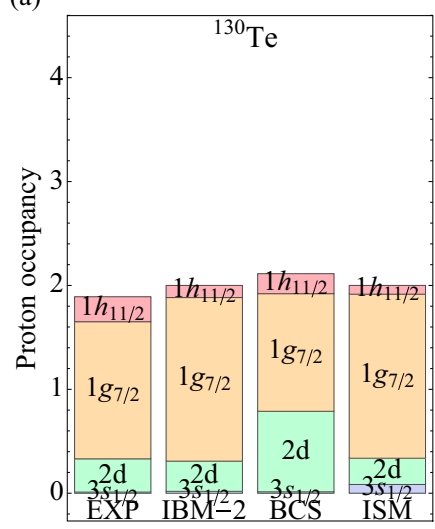

(b)

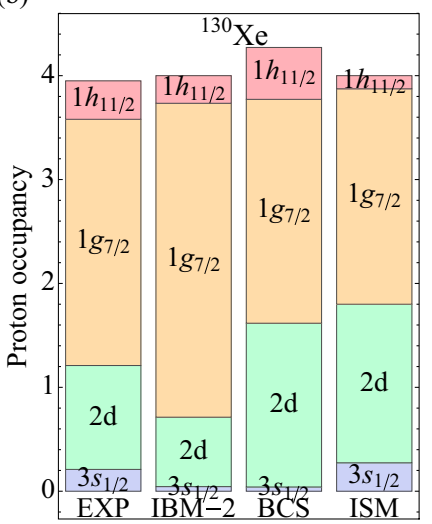

(c)

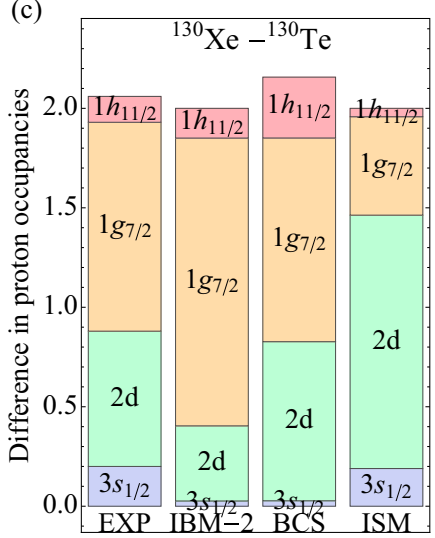

FIG. 7. IBM-2 occupations in (a) ${ }^{130} \mathrm{Te}$, (b) ${ }^{130} \mathrm{Xe}$, and (c) their change for protons, compared with experimental [5], BCS [41], and ISM [8] results. 
(a)

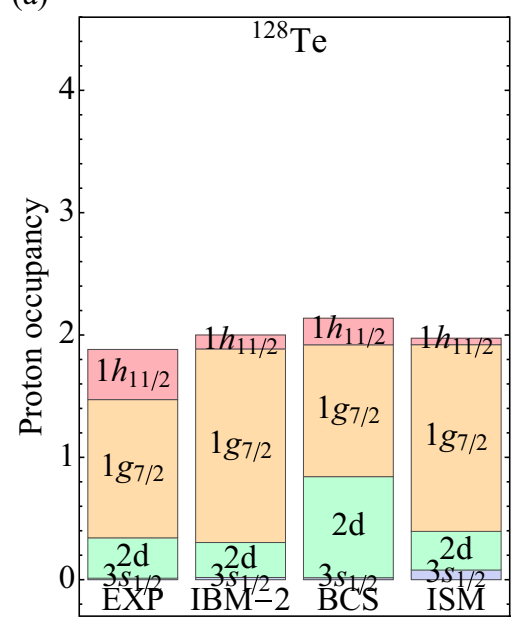

(b)

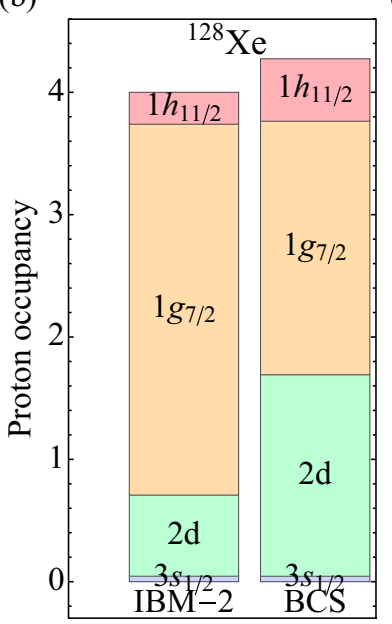

(c)

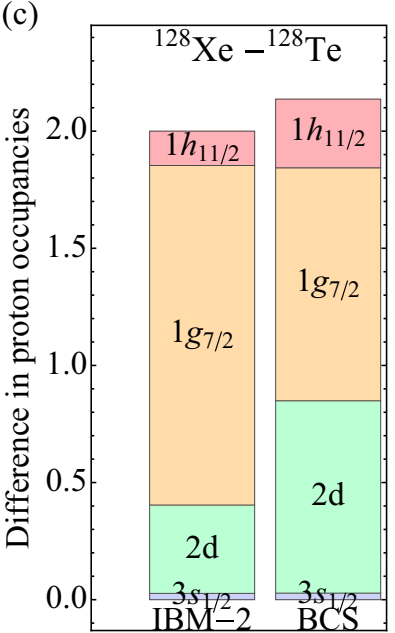

FIG. 8. Same as Fig. 7 for ${ }^{128} \mathrm{Te}$ and ${ }^{128} \mathrm{Xe}$.

are shown in Fig. 10 for neutrons and Fig. 12 for protons, while those obtained with Set II are shown in Fig. 11 for neutrons and Fig. 13 for protons. By using Set II we are studying the effect that assuming subshell closure at $Z=64$ has on occupancies.

The two sets of single particle energies are very different and thus very different results for occupancies are expected. For neutrons (Figs. 10 and 11), both calculations predict the change to be largely due to $2 f$ orbitals. Set I shows also a large contribution for $3 p$ orbitals, while Set II is giving a strong contribution from the $1 i_{13 / 2}$ orbital. Both calculations show smaller contributions from the $2 f_{5 / 2}$ and $1 h_{9 / 2}$ orbitals. In the case of proton occupancy obtained using Set I (Fig. 12), the change is predicted to be dominated by the $1 g_{7 / 2}$ orbital with smaller contributions from the $1 h_{11 / 2}$ and $2 d$ orbitals, while Fig. 13, obtained using Set II, shows that the change is dominated by the $1 h_{11 / 2}$ orbital, with a smaller contribution from the $1 g_{7 / 2}$ orbital. Overall, assuming the subshell closure at $Z=64$, the highest- $\ell$ orbitals, $1 i_{13 / 2}$ for neutrons and $1 h_{11 / 2}$ for protons, become more filled. Also in this case there are experiments in progress and the preliminary experimental results for change in neutron occupancy in these nuclei [4] suggest very complex rearrangement of nucleons. It will be interesting to compare the obtained results with experiments once reliable data are available.

\section{F. Sensitivity of occupancies to the choice of single particle energies}

In Ref. [14] a study of the sensitivity of double- $\beta$ decay nuclear matrix elements to the choice of single particle energies was already performed, where a comparison between the results using energies extracted from experiments were compared with those obtained using the energies from a calculation in a Woods-Saxon well. The impact on the double- $\beta$ decay nuclear matrix elements due to the differences between the single particle energies used in this work and those of Ref. [14] will be discussed in a forthcoming publication. (a)

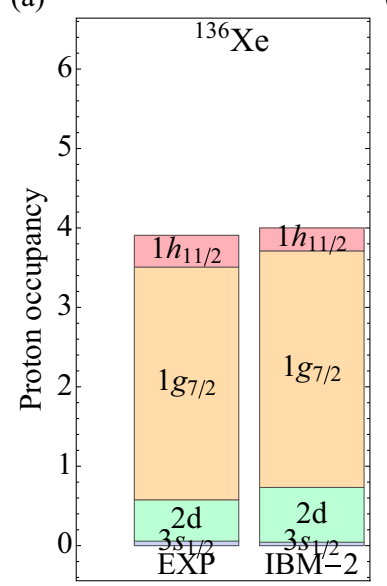

(b)

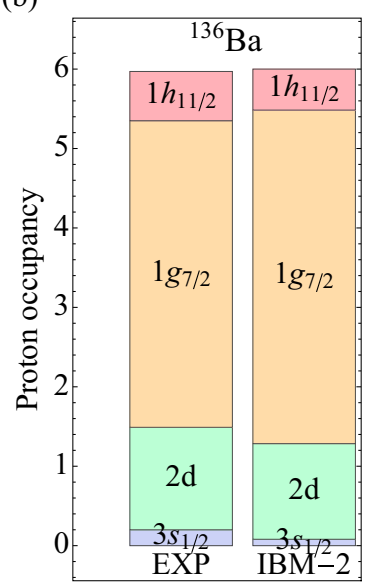

(c)

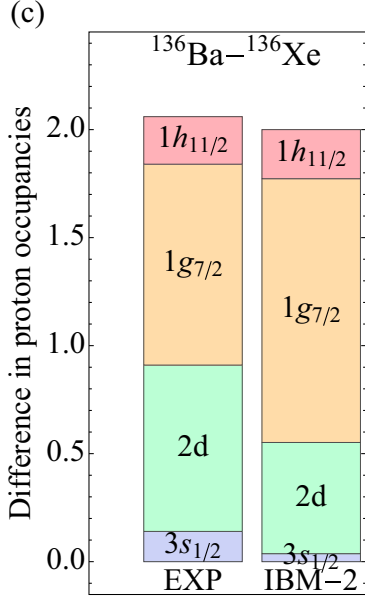

(d)

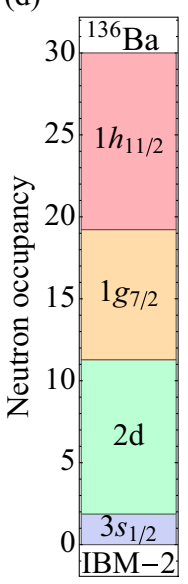

FIG. 9. IBM-2 occupations in (a) ${ }^{136} \mathrm{Xe}$, (b) ${ }^{136} \mathrm{Ba}$, and their change (c) for protons, and (d) predicted IBM-2 occupations in ${ }^{136} \mathrm{Ba}$ for neutrons. 
(a)

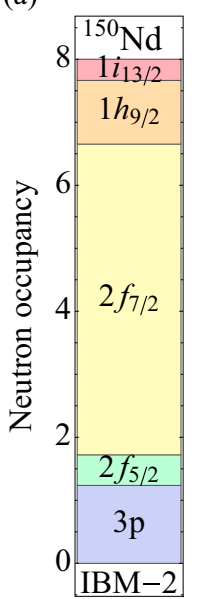

(b)

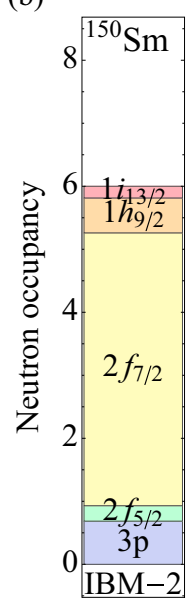

(c)

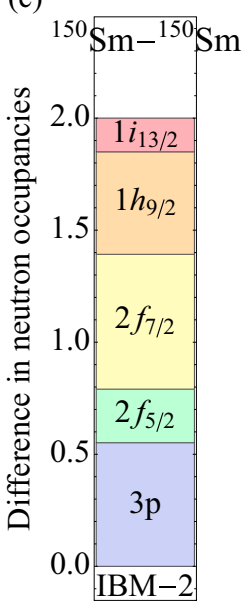

FIG. 10. IBM-2 occupations in (a) ${ }^{150} \mathrm{Nd}$, (b) ${ }^{150} \mathrm{Sm}$, and (c) their change for neutrons using the single particle energies of Set I.

Here we focus on studying how the occupancies are affected when using different sets of single particle energies. To this end we have considered seven different sets of single particle energies obtained from a calculation using a Woods-Saxon potential with fixed depth and varying radius [45]. These sets have been used to calculate the proton and neutron occupancies in ${ }^{130} \mathrm{Te}$, which are shown in Fig. 14 , and in ${ }^{150} \mathrm{Nd}$, which are shown in Fig. 15.

Figures 14(a) and 14(b) show the evolution of the WoodsSaxon single particle energies in the shell 50-82 for neutrons and protons, respectively. Figures $14(\mathrm{c})$ and 14(d) show the calculated occupancies. As we can see from them, the occupancies are rather stable for neutrons, because in this case, ${ }^{130} \mathrm{Te}$, neutrons almost fill the shell $50-82$. However, for protons an interchange between $2 d_{5 / 2}$ and $1 g_{7 / 2}$ carrying most of the occupation is seen, corresponding to the exchange of these single particle levels in Fig. 14(b). In Fig. 15,

(a)

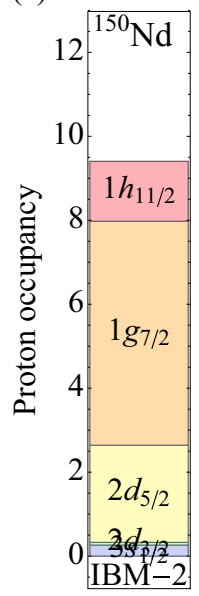

(b)

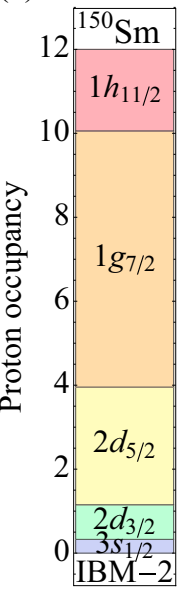

(c)

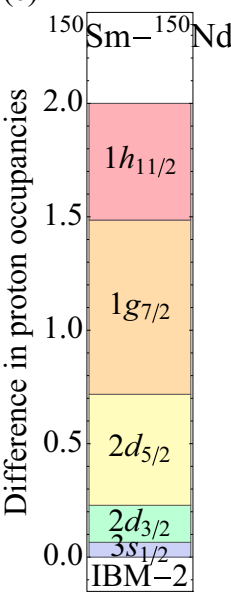

FIG. 12. IBM-2 occupations in (a) ${ }^{150} \mathrm{Nd}$, (b) ${ }^{150} \mathrm{Sm}$, and (c) their change for protons using the single particle energies of Set I.

corresponding to ${ }^{150} \mathrm{Nd}$, the picture is different because now both protons and neutrons are filling the shells 50-82 and $82-126$ partially. In this case the neutron occupancy is dominated by the $2 f_{7 / 2}$ orbital, followed by the orbitals $3 p_{3 / 2}$ and $1 h_{9 / 2}$, which exchange their dominance according the exchange in their single particle energies. For protons the results are similar to what happens in ${ }^{130} \mathrm{Te}$, the occupancy is dominated by the orbitals $2 d_{5 / 2}$ and $1 g_{7 / 2}$, whose dominance is interchanged as their single particle energies are exchanged. Preliminary studies on the double- $\beta$ decay matrix elements indicate that their values remains unaltered for ${ }^{130} \mathrm{Te}$, but for ${ }^{150} \mathrm{Nd}$ the Gamow-Teller matrix elements show some change. This suggests that those nuclei with few valence nucleons are not as affected by the change in single particle energies as those ones with several valence nucleons. Detailed analysis of this dependence will be performed in a subsequent publication once the experimental data of occupancies are available.

(a)

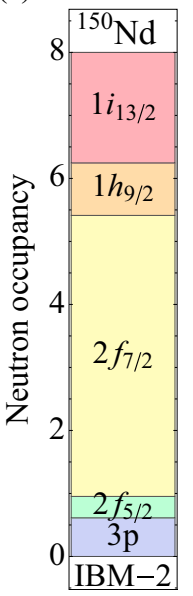

(b)

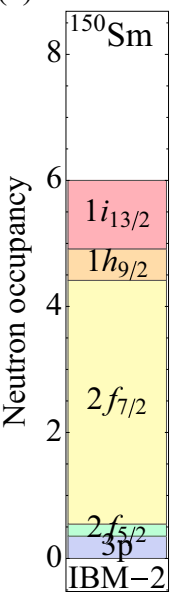

(c)

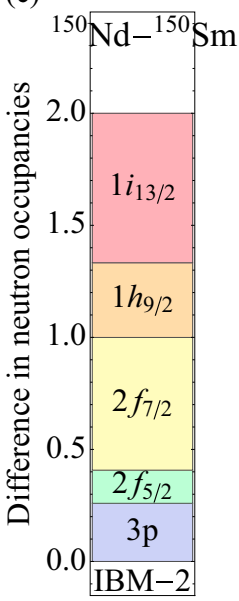

FIG. 11. IBM-2 occupations in (a) ${ }^{150} \mathrm{Nd}$, (b) ${ }^{150} \mathrm{Sm}$, and (c) their change for neutrons using the single particle energies of Set II.

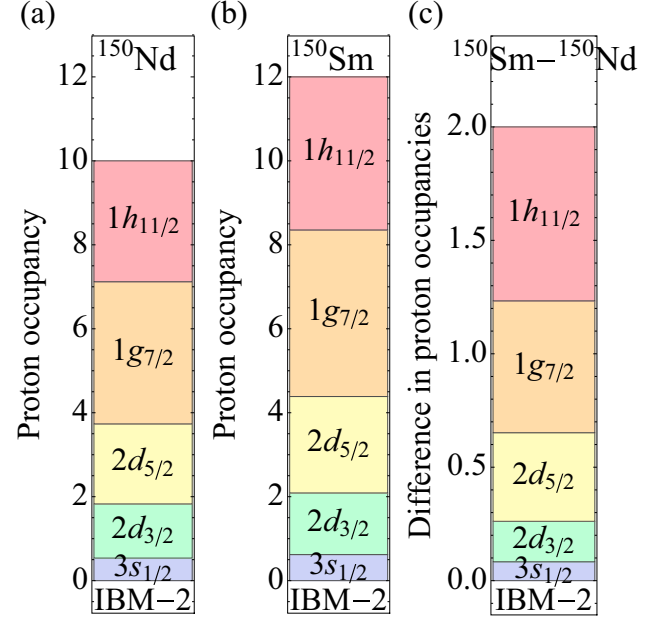

FIG. 13. IBM-2 occupations in (a) ${ }^{150} \mathrm{Nd}$, (b) ${ }^{150} \mathrm{Sm}$, and (c) their change for protons using the single particle energies of Set II. 
(a)

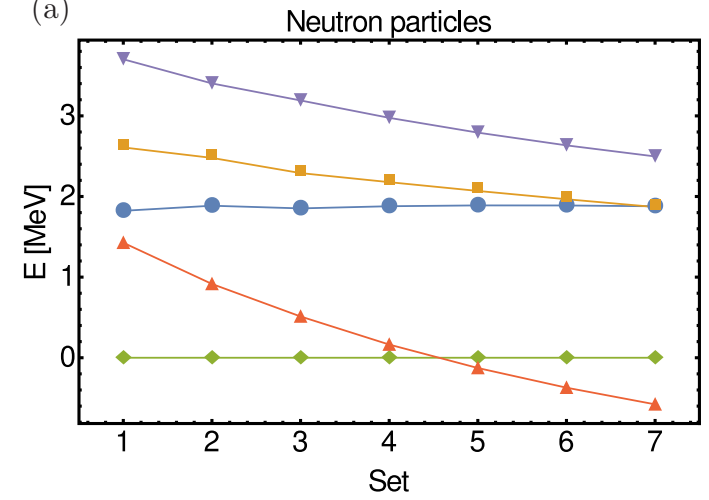

(b)

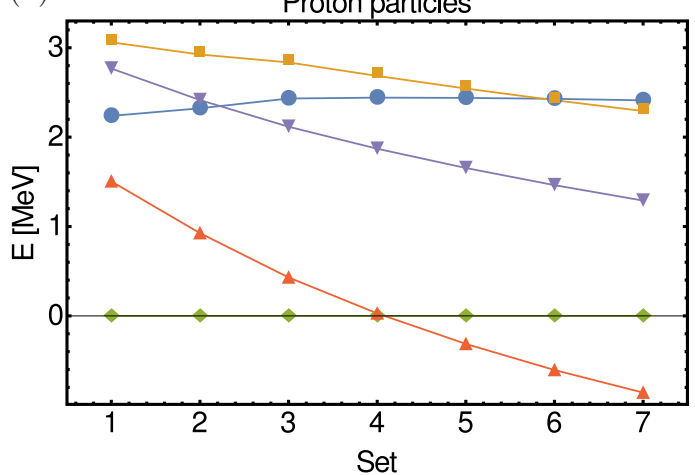

(c)

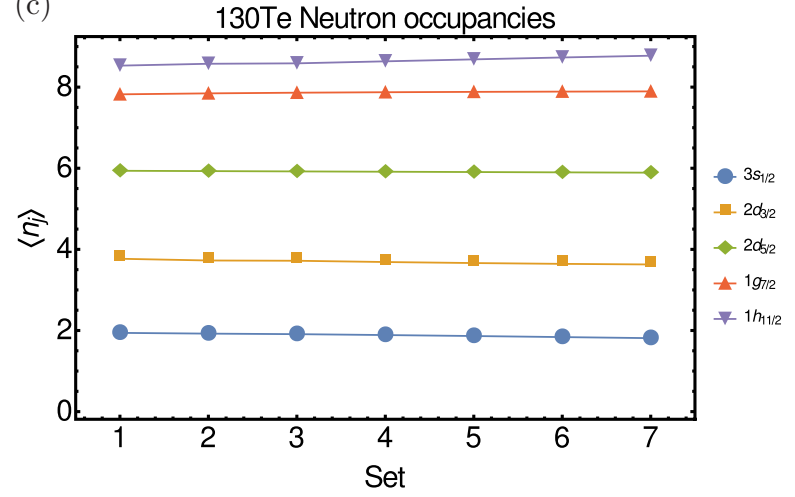

(d)

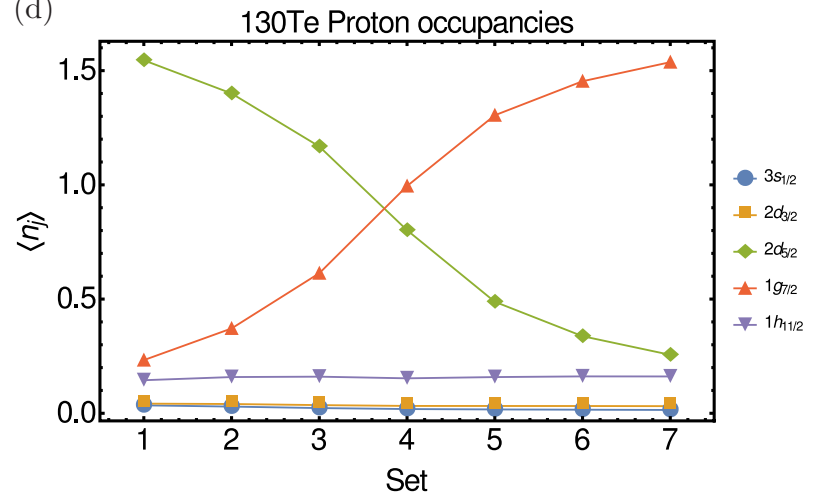

FIG. 14. (a) Different single particle energies for neutrons. (b) Different single particle energies for protons. (c) Evolution of occupancies for neutrons. (d) Evolution of occupancies for protons.

(a)

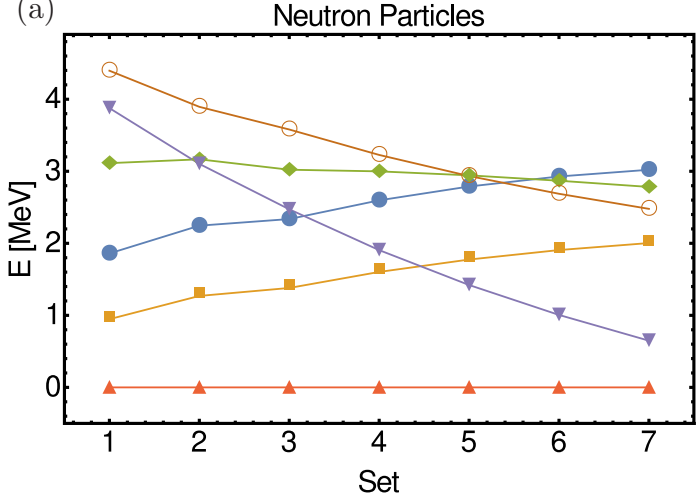

(b)

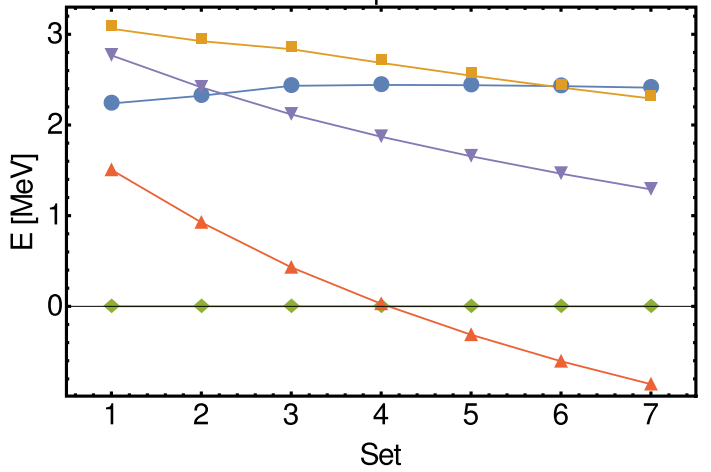

(c)

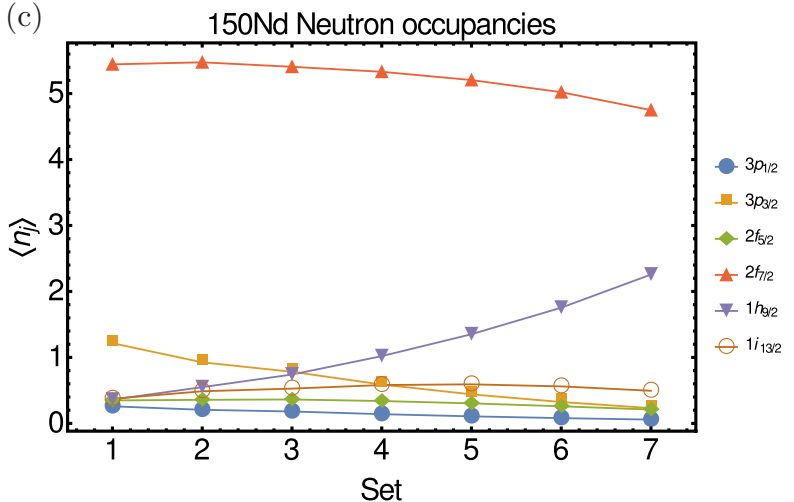

(d)

150Nd Proton occupancies

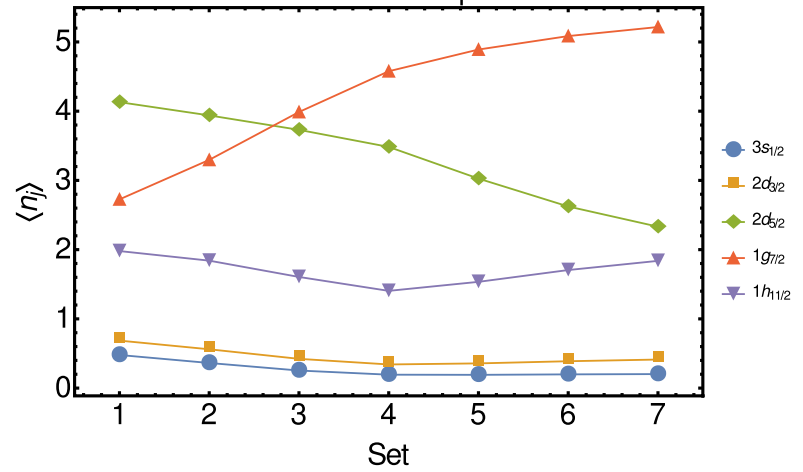

FIG. 15. Same as Fig. 14. 


\section{CONCLUSIONS}

In this article we report a new method to calculate the occupancies of single particle levels in the context of the microscopic interacting boson model IBM-2. This method depends on the structure coefficients of the $S$ and $D$ pair creation operators. In this work, they were obtained by diagonalizing a SDI in the space of two particles and assuming that these pairs create the energetically lowest $0^{+}$and $2^{+}$two-nucleon states. The calculation includes the choice of single particle energies, which in this case are taken from experimental data. The needed SDI strength is fitted to reproduce the $2_{1}^{+}-0_{1}^{+}$ energy difference in the corresponding two-valence-nucleon nuclei. Then the IBM-2 wave functions, which reproduce well the spectroscopic data, are used to calculate the expectation value of the number operator to obtain the occupancies.

We have applied this method to calculate the ground-state occupancies of valence protons and neutrons of double- $\beta$ decay nuclei with $A=76,100,128,130,136$, and 150 with comparison to available experimental data and to QRPA at BCS level and ISM calculations. IBM-2 gives reasonable agreement without any adjustments of the single particle energies for the cases where comparison with experiments are possible. In the cases where there are no experimental data yet available $\left({ }^{128} \mathrm{Xe}\right.$ for protons and neutrons, ${ }^{136} \mathrm{Ba}$ for neutrons, and ${ }^{150} \mathrm{Nd}$ and ${ }^{150} \mathrm{Sm}$ for neutrons and protons), IBM-2 makes predictions that can be tested with future experiments. These predictions differ from BCS calculations. Particularly interesting is the case of ${ }^{150} \mathrm{Nd}-{ }^{150} \mathrm{Sm}$. The predictions here are unique to IBM-2, because the ISM calculation for these nuclei cannot be performed due to the large model space and QRPA calculations are difficult to perform due to the instabilities of the spherical-deformed transition.

\section{ACKNOWLEDGMENTS}

This work was supported by the Academy of Finland (Suomen Akatemia) (Project No. 266437) and Chilean Ministry of Education (Fondo Nacional de Desarrollo Científico y Tecnológico) (Grant No. 1150564). The authors thank Professor F. Iachello for valuable discussions. Also, we would like to thank the Referee for useful comments and remarks.
[1] J. P. Schiffer, S. J. Freeman, J. A. Clark, C. Deibel, C. R. Fitzpatrick, S. Gros, A. Heinz, D. Hirata, C. L. Jiang, B. P. Kay, A. Parikh, P. D. Parker, K. E. Rehm, A. C. C. Villari, V. Werner, and C. Wrede, Phys. Rev. Lett. 100, 112501 (2008).

[2] B. P. Kay et al., Phys. Rev. C 79, 021301(R) (2009).

[3] B. P. Kay, T. Bloxham, S. A. McAllister, J. A. Clark, C. M. Deibel, S. J. Freedman, S. J. Freeman, K. Han, A. M. Howard, A. J. Mitchell, P. D. Parker, J. P. Schiffer, D. K. Sharp, and J. S. Thomas, Phys. Rev. C 87, 011302(R) (2013).

[4] D. K. Sharp, AIP Conf. Proc. 1686, 020021 (2015); http://medex15.utef.cvut.cz/talks/Sharp.pdf.

[5] J. P. Entwisle et al., Phys. Rev. C 93, 064312 (2016).

[6] O. Moreno, E. Moya de Guerra, P. Sarriguren, and A. Faessler, Phys. Rev. C 81, 041303(R) (2010).

[7] J. Suhonen and O. Civitarese, Nucl. Phys. A 924, 1 (2014).

[8] A. Neacsu and M. Horoi, Phys. Rev. C 91, 024309 (2015).

[9] J. Engel, J. Phys. G: Nucl. Part. Phys. 42, 034017 (2015).

[10] F. Iachello and A. Arima, The Interacting Boson Model (Cambridge University Press, Cambridge, England, 1987).

[11] J. Kotila and F. Iachello, Phys. Rev. C 85, 034316 (2012).

[12] J. Kotila and F. Iachello, Phys. Rev. C 87, 024313 (2013).

[13] J. Kotila, J. Barea, and F. Iachello, Phys. Rev. C 89, 064319 (2014).

[14] J. Barea and F. Iachello, Phys. Rev. C 79, 044301 (2009).

[15] J. Barea, J. Kotila, and F. Iachello, Phys. Rev. Lett. 109, 042501 (2012).

[16] J. Barea, J. Kotila, and F. Iachello, Phys. Rev. C 87, 014315 (2013).

[17] J. Barea, J. Kotila, and F. Iachello, Phys. Rev. C 87, 057301 (2013).

[18] J. Barea, J. Kotila, and F. Iachello, Phys. Rev. C 91, 034304 (2015).

[19] J. Kotila, J. Barea, and F. Iachello, Phys. Rev. C 91, 064310 (2015).
[20] J. Barea, J. Kotila, and F. Iachello, Phys. Rev. D 92, 093001 (2015)

[21] J. Menéndez, A. Poves, E. Caurier, and F. Nowacki, Phys. Rev. C 80, 048501 (2009).

[22] J. Suhonen and O. Civitarese, Phys. Lett. B 668, 277 (2008).

[23] F. Šimkovic, A. Faessler, and P. Vogel, Phys. Rev. C 79, 015502 (2009).

[24] A. Arima, T. Otsuka, F. Iachello, and A. Talmi, Phys. Lett. B 66, 205 (1977).

[25] T. Otsuka, A. Arima, and F. Iachello, Nucl. Phys. A 309, 1 (1978)

[26] I. Talmi, Nucl. Phys. A 172, 1 (1971).

[27] A. Klein and M. Valliéres, Phys. Lett. B 98, 5 (1981).

[28] O. Scholten, Phys. Rev. C 28, 1783 (1983).

[29] O. Scholten, Prog. Part. Nucl. Phys. 14, 189 (1985).

[30] N. Yoshinaga, T. Mizusaki, A. Arima, and Y. D. Devi, Prog. Theor. Phys. Suppl. 125, 65 (1996).

[31] S. Pittel, P. D. Duval, and B. R. Barrett, Ann. Phys. (NY) 144, 168 (1982).

[32] A. Frank and P. Van Isacker, Phys. Rev. C 26, 1661 (1982).

[33] P. O. Lipas et al., Nucl. Phys. A 509, 509 (1990).

[34] W. J. Baldridge, Phys. Rev. C 18, 530 (1978).

[35] P. Kleinheinz, R. Broda, P. J. Daly, S. Lunardi, M. Ogawa, and J. Blomquist, Z. Phys. A 290, 279 (1979).

[36] M. Ogawa, R. Broda, K. Zell, P. J. Daly, and P. Kleinheinz, Phys. Rev. Lett. 41, 289 (1978).

[37] Y. Nagai, J. Styczen, M. Piiparinen, P. Kleinheinz, D. Bazzacco, P. V. Brentano, K. O. Zell, and J. Blomqvist, Phys. Rev. Lett. 47, 1259 (1981).

[38] N. R. Walet, P. Stoop, and P. W. M. Glaudemans, Z. Phys. A 332, 9 (1989).

[39] S. W. Yates, R. Julin, P. Kleinheinz, B. Rubio, L. G. Mann, A. Henry, W. Stöffl, D. J. Decman, and J. Blomqvist, Z. Phys. A 324, 417 (1986). 
[40] L. G. Mann, D. J. Decman, H. E. Martz, T. N. Massey, G. L. Struble, P. Kleinheinz, H. J. Scheerer, D. G. Burke, and G. Kajrys, Z. Phys. A 337, 301 (1990).

[41] J. Suhonen and O. Civitarese, Nucl. Phys. A 847, 207 (2010).

[42] J. Hyvärinen and J. Suhonen, Phys. Rev. C 91, 024613 (2015); (private communication).
[43] J. Menéndez, A. Poves, E. Caurier, and F. Nowacki, J. Phys.: Conf. Ser. 312, 072005 (2011).

[44] P. D. Duval, D. Goutte, and M. Vergnes, Phys. Lett. B 124, 297 (1983).

[45] S. Cwiok, J. Dudek, W. Nazarewicz, J. Skalski, and T. Werner, Comput. Phys. Commun. 46, 379 (1987). 